\title{
IMPLANTATION COCHLÉAIRE ET MALFORMATION DE L'OREILLE INTERNE
}

\author{
G. BESBES, S. KHARRAT, S. TABABI, H. BEN RHOUMA, R. ZAININE, \\ N. BELTAIEF, E. MENIF*, S. SAHTOUT. \\ SERVICE ORL \\ *SERVICE DE RADIOLOGIE \\ HÔPITAL LA RABTA.
}

\begin{abstract}
RESUME
Les indications de l'implant cochléaire se sont élargies depuis 1995. La plupart des candidats à l'implant sont des enfants sourds profonds congénitaux. L'implant peut actuellement se discuter dans certains cas particuliers notamment chez des enfants présentant une malformation de l'oreille interne (vésicule unique, malformation de type Mondini, Mondini-like, dilatation de l'aqueduc du vestibule). Les risques sont chirurgicaux et infectieux : geyser à la cochléostomie, paralysie faciale, otoliquorrhée secondaire et méningite.

Nous rapportons le cas d'une fille âgée de 4 ans et 8 mois qui a présenté suite à un traumatisme minime une surdité brutale totale bilatérale qui n'a pas récupéré sous traitement médical. L'audiométrie avait confirmé la surdité bilatérale et l'examen orthophonique a retrouvé un langage oral bien conservé. Une imagerie a été pratiquée mettant en évidence une dilatation bilatérale des aqueducs du vestibule et des sacs endolymphatiques avec un aspect normal de la cochlée et des canaux semi-circulaires.

La patiente a bénéficiée d'une implantation cochléaire gauche avec des suites simples et un excellent résultat à 2 ans. Mots-clés : Implant cochléaire, malformation de l'oreille interne.
\end{abstract}

In recent years, many studies have clearly documented the effectiveness of cochlear implantation in children. Since cochlear malformations have been reported to occur in approximately $20 \%$ of children with congenital sensorineural hearing loss, the success of implantation in this group of patients is of special interest. Although inner ear malformation was once regarded as a contraindication for cochlear implantation, studies at different centers have proved this treatment not only to be feasible.

Enlarged vestibular aqueduct (EVA) is the most common, radiographically evident abnormality of the inner ear in association with congenital or early onset sensorineural hearing loss. It can occur in isolation or in association with other inner ear malformations, such as the Mondini malformation. However, congenital anomalies of the inner ear are associated with an increased incidence of both cerebrospinal fluid (CSF) leak at cochleostomy and aberrant facial nerve course, which can lead to postoperative CSF leak, meningitis, and facial nerve complications in association with cochlear implantation. We report the case of a 4 year old girl presenting with total deafness after minor head trauma.

Radiological abnormality was an isolated vestibular aqueduct enlargement in both ears. Audiological explorations find bilateral total deafness. The oral language was preserved.

She was implanted for her left ear with excellent result.

Keywords : Cochlear implant, malformation of inner ear.

\section{INTRODUCTION}

Les indications de l'implant cochléaire se sont élargies depuis 1995. La plupart des candidats à l'implant sont des enfants sourds profonds congénitaux pour lesquels la mise en place de l'implant doit être très précoce afin d'optimiser le développement de la perception et du langage. L'implant peut actuellement se discuter dans certains cas particuliers, chez des enfants ayant des pathologies associées, en cas de malformations d'oreille interne ou encore de surdités sévères ou profondes.
Nous rapportons un cas d'implantation cochléaire chez un enfant porteur d'un syndrome de l'aqueduc du vestibule large.

\section{OBSERVATION}

Fille, âgée de 4 ans 8 mois sans antécédents pathologiques notables (en particulier absence de notion de surdité familiale ou d'infection néonatale) ayant présenté

7 mois auparavant un traumatisme crânien minime suite à un accident domestique (chute d'une hauteur d'un 
mètre) occasionnant une surdité totale bilatérale. L'otoscopie était normale. Les potentiels évoqués auditifs ont confirmé la surdité bilatérale et profonde. L'examen orthophonique avait montré un langage oral bien conservé. La patiente a bénéficié d'un scanner des rochers montrant une dilatation bilatérale de l'aqueduc du vestibule avec un aspect normal de la cochlée, des canaux semicirculaires et du vestibule, un aspect normal de la caisse et de la mastoïde avec absence de trait de fracture (fig. 1 et 2). Un complément d'imagerie par résonnance magnétique a été pratiqué confirmant la dilatation des aqueducs du vestibule (fig. 3 et 4). Une consultation génétique a été réalisée éliminant un syndrome de Pendred.

La patiente a été opérée avec mise en place d'un implant cochléaire gauche sans incidents per opératoires, avec au contrôle radiologique une électrode en place (fig. 5). Les suites opératoires ont été simples avec un excellent résultat, une bonne intégration et de bons résultats scolaires avec un langage oral bien conservé. Le recul est de 3 ans.
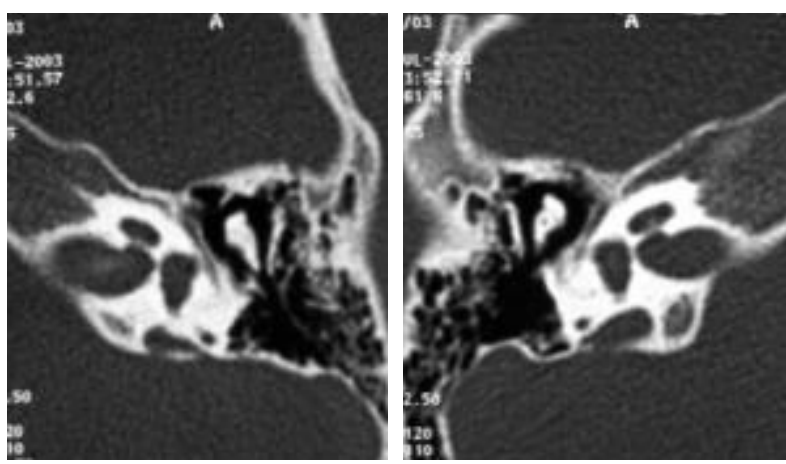

Fig. 1 et 2 : TDM des rochers en coupes axiales montrant une dilatation bilatérale de l'aqueduc du vestibule avec un aspect normal de la cochlée, des canaux semi circulaires, du vestibule, de la caisse et de la mastoïde avec absence de trait de fracture.
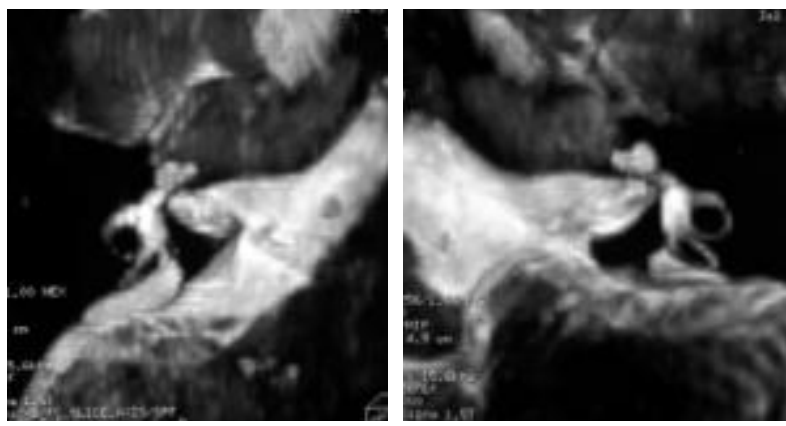

Fig. 3 et 4 : IRM en coupes axiales, en séquence pondérée T2 montrant une dilatation du sac endolymphatique

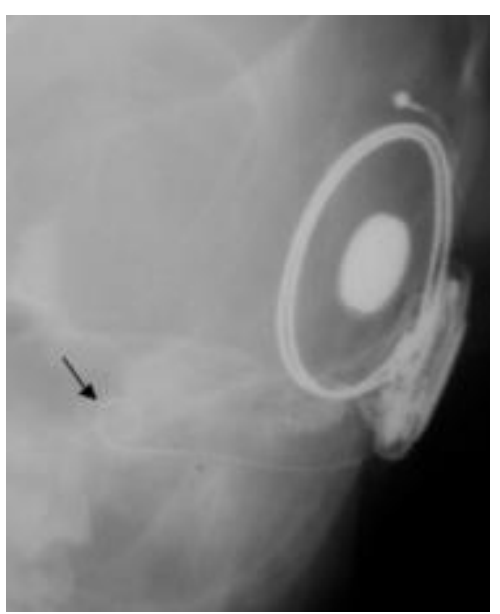

Fig. 5 : Radiographie standard : électrode en place

\section{DISCUSSION}

Les malformations de l'oreille interne représentent approximativement $20 \%$ des étiologies des surdités neurosensorielles congénitales (1).

Auparavant, les malformations de l'oreille interne étaient considérées comme des contre-indications à l'implantation cochléaire $(2,3)$; ceci est du essentiellement à des bases histologiques prouvant la rareté du tissu nerveux au niveau des oreilles malformées $(4,5)$.

Le premier cas d'implantation cochléaire sur oreille interne malformée a été rapporté en 1983 par Mangabeiria et Albernaz mais ils ont découvert cette malformation en per-opératoire (6). Et depuis, plusieurs patients présentant une malformation d'oreille interne ont été implantés avec succès par de nombreuses équipes $(2,3)$. Ces malformations peuvent être sévères (vésicule unique, malformation de type Mondini, Mondini-like) ou plus modérées (dilatation de l'aqueduc du vestibule).

En 1997, Luntz a rapporté les résultats de 10 enfants sourds pré-linguaux, porteurs d'une malformation congénitale d'oreille interne (7). Les malformations comprenaient des vésicules uniques, des segmentations cochléaires incomplètes (Mondini ou Mondini-like) et une dilatation de l'aqueduc du vestibule. Après 30 mois d'utilisation, $75 \%$ des patients ont montré des capacités de reconnaissance de mots en listes ouvertes. Selon la littérature, les malformations cochléaires sont compatibles avec l'implantation cochléaire à l'exception des aplasies labyrinthiques et des hypoplasies du conduit auditif interne $(2,3)$. En cas de cophose avec conduits auditifs internes étroits au scanner, I'IRM et la stimulation du nerf auditif sont indispensables pour éliminer une agénésie du nerf auditif qui contre indiquerait la chirurgie.

Actuellement, la seule contre-indication à l'implantation cochléaire en dehors de l'absence du nerf cochléaire est la malformation de Michel ou l'aplasie labyrinthique totale $(8,9,10)$. 
Le syndrome de l'aqueduc de vestibule large (SAVL) représente la malformation de l'oreille interne la plus fréquente, radiologiquement détectable. II est souvent bilatéral, et se voit le plus fréquemment chez les sujets de sexe féminin (sex-ratio 2/3). II est souvent associé à d'autres types de malformations de l'oreille interne surtout avec la partition incomplète type 2 (Mondini) (8). L'évolution se fait progressivement vers la surdité neurosensorielle (11).

II peut être associé même s'il est isolé à des déhiscences osseuses intra-cochléaires infra radiologiques pouvant causer la surdité en cas de traumatisme crânien même minime (12).

Comme les autres malformations de l'oreille interne, ce syndrome est associé à une grande incidence de survenue de geyzer lors de la cochléostomie et de procidence du nerf facial ce qui explique le risque accru de fuite de LCR, de méningite et de complications en rapport avec le nerf facial (13).

Le geyzer au moment de la cochléostomie survient dans 15 à $100 \%$ des cas (14), et est dû soit à un sac endolymphatique dilaté soit à l'absence du modiolus et/ou des septums interscalaires pouvant passer inaperçus au scanner mais détectables en IRM avec fast spin écho. Cependant, il n'y a pas de corrélation entre l'imagerie et les découvertes per-opératoires.

Par ailleurs, il est important de signaler que le geyzer est moins important quantitativement lors du SAVL que lors des autres malformations : fuite à faible débit ou « oozer» (8). Cette fuite est souvent facilement contrôlable en peropératoire mais le risque méningitique reste élevé même plusieurs mois après l'implantation (14).

Une vaccination anti-hémophilus et anti-pneumocoque est indispensable en préopératoire ainsi qu'un traitement péri-opératoire diminuant la pression intra labyrinthique (Mannitolß).

Les anomalies de trajet du nerf facial sont assez fré- quentes au cours des malformations de l'oreille interne, retrouvées dans 16 à $32 \%$ des cas $(16,17)$. La portion tympanique se situe en position plus antérieure, inférieure et latérale impliquant un risque important de paralysie faciale postopératoire d'où l'importance d'un monitorage systématique du nerf facial $(12,16)$.

L'incidence de stimulation du nerf facial par l'implant cochléaire en post-opératoire (estimée à 11\%) est plus importante que chez les sujets indemnes de malformation, ceci est du très probablement au trajet anormal du nerf dans ses portions tympanique et mastoïdienne (16). Il existe par ailleurs des problèmes techniques en rapport avec la difficulté d'insertion de l'électrode qui sera incomplète dans $21 \%$ des cas chez les patients porteurs de malformations de l'oreille interne (16). En ce qui concerne le SAVL, ce problème devient sérieux en cas d'association avec d'autres malformations. Ce défaut d'insertion d'électrode risque d'affecter les performances auditives mais généralement les résultats sont satisfaisants chez la majorité des patients $(13,17)$.

\section{CONCLUSION}

Les patients porteurs de malformations de l'oreille interne étaient longtemps privés de la chance d'avoir un implant cochléaire. Actuellement, hormis ceux porteurs d'une malformation de Michel, ces patients peuvent bénéficier de cette implantation. Les risques opératoires sont certes présents et réels mais les résultats sont fort encourageants et nous poussent à avancer encore dans cette perspective fort prometteuse.

\section{REFERENCES}

1. Sennaroglu L, Sarac S, Ergin T. Surgical results of cochlear implantation in malformed cochlea. Otol Neurotol. 2006; 27(5):615-23.

2. Loundon N, Rouillon I, Munier N, Marlin S, Roger G, Garabedian EN. Cochlear implantation in children with internal ear malformations. Otol Neurotol. 2005;26(4):668-73.

3. Weber BP, Dillo W, Dietrich B. Pediatric cochlear implantation in cochlear malformations. Am J Otol 1998; 19 :747- 53.

4. Monsell EM, Jackler RK, Motta G. Congenital malformations of the inner ear: histologic findings in five temporal bones. Laryngoscope 1987; 97:18- 24

5. Schmidt JM. Cochlear neuronal populations in developmental defects of the inner ear. Acta Otolaryngol (Stockh) 1985; 99:14-20.

6. Mangabeira-Albernaz PL. The Mondini dysplasia from early diagnosis to cochlear implant. Acta Otolaryngol 1983; 95: 627-31.

7- Luntz M, Balkany T, Telischi FF, Hodges AV. Surgical techniques for cochlear implantation of the malformed inner ear. Am J Otol. 1997;18(6):S66.

8- Doherty JK., Luxford WM. Cochleostomy management in patients with enlarged vestibular aqueduct receiving cochlear implants. Operative Techniques in Otolaryngology 2005; 16: 82-85.

9- Miyamoto RT. Cochlear implantation for cochlear dysplasia. Operative Techniques in Otolaryngology 2005; 16: 121-124.
10- Arnoldner C., Baumgartner WD. Audiological performance after cochlear implantation in children with inner ear malformations. international Journal of Pediatric Otorhinolaryngology 2004; 68 : 457-467

11- Valvassori GE, Clemis JD. The large vestibular aqueduct syndrome. Laryngoscope 1978; 88: 723-728.

12- Jackler RK, Luxford WM, House WF. Congenital malformations of the inner ear: A classification based on embryogenesis. Laryngoscope 1987 (40); 97 : 214.

13- Slattery WH., Luxford WM. Cochlear implantation in the congenital malformed cochlea. Laryngoscope 1995; 105: 1184-1187.

14- Mylanus EA, Rotteveel LJ, Leeuw RL. Congenital malformation of the inner ear and pediatric cochlear implantation. Otol Neurotol. 2004; 25(3):308-17. 15- Kim LS, Jeong SW, Huh MJ, Park YD. Cochlear implantation in children with inner ear malformations. Ann Otol Rhinol Laryngol. 2006;115(3):205-14.

16- Buchman CA, Copeland BJ, Yu KK, Brown CJ, Carrasco VN, Pillsbury HC 3rd. Cochlear implantation in children with congenital inner ear malformations. Laryngoscope. 2004;114(2):309-16.

17- Miyamoto RT, Bichey BG, Wynne MK, Kirk KI. Cochlear implantation with large vestibular aqueduct syndrome. Laryngoscope. 2002;112(7):1178-82. 